\title{
Discrete Current Control Strategy of Permanent Magnet Synchronous Motors
}

\author{
Yan Dong, ${ }^{1}$ Kai Jing, ${ }^{1}$ Hexu Sun, ${ }^{1,2}$ and Yi Zheng' \\ ${ }^{1}$ School of Control Science and Engineering of Hebei University of Technology, Tianjin 300130, China \\ ${ }^{2}$ Hebei University of Science and Technology, Shijiazhuang 050018, China \\ Correspondence should be addressed to Hexu Sun; hxsun@hebust.edu.cn
}

Received 1 July 2013; Revised 22 September 2013; Accepted 28 September 2013

Academic Editor: Baocang Ding

Copyright (c) 2013 Yan Dong et al. This is an open access article distributed under the Creative Commons Attribution License, which permits unrestricted use, distribution, and reproduction in any medium, provided the original work is properly cited.

A control strategy of permanent magnet synchronous motors (PMSMs), which is different from the traditional vector control (VC) and direct torque control (DTC), is proposed. Firstly, the circular rotating magnetic field is analyzed on the simplified model and discredited into stepping magnetic field. The stepping magnetomotive force will drive the rotor to run as the stepping motor. Secondly, the stator current orientation is used to build the control model instead of rotor flux orientation. Then, the discrete current control strategy is set and adopted in positioning control. Three methods of the strategy are simulated in computer and tested on the experiment platform of PMSM. The control precision is also verified through the experiment.

\section{Introduction}

The permanent magnet synchronous motors (PMSMs) have become the popular AC motors and are used in various situations for their advantages of high efficiency, power factor, small size, and avoidance of exciting current. As servo motors, PMSMs are usually controlled with two methods, that is, vector control (VC) by flux orientation and direct torque control (DTC).

VC was put forward in 1971 for asynchronous motor by German engineer Blaschke [1], which was used in PMSM soon afterwards. Generally, the theory is to keep the d component of stator current being 0 in rotor flux reference frame and the torque will be proportionate to the q component of stator current which leads the constant rotor flux by $90^{\circ}$. It is good at torque responding and speed accuracy, but the decoupling of flux and torque needs more focus to design regulator for both. The robustness will be vulnerable [2].

DTC is proposed by Professor Depenbrock in 1985 [3], which is used to directly control the flux and torque by selecting proper voltage vector. This method avoids the decoupling and is simpler than VC, but the torque ripple cannot be avoided which will weaken the dynamic characteristic $[4,5]$.

Both methods are based on rotor flux which needs to be tested by an observer or to be controlled with other variables $[6,7]$. This paper proposes a strategy based on stator current frame and uses the discrete stator current to control the motor. By using this strategy, the motor will run step by step, and it not only reflects the simply structure and large capacity of PMSM but also provides the advantages of stepping motor such as digital control, discrete operation, and nonaccumulating error. The proposed strategy is a novel control method on PMSMs with simple control structure as compared with the above two classical methods. The wide application prospects and the deep research of it will promote the development of drive technology.

\section{Discretization of Circular Rotating Magnetic Field}

2.1. Stator Model of PMSM. In PMSM, distributed winding, which is used in normal AC motor, is often coiled as shown in Figure 1. Figure 1 shows two structures of 2-pole, 24-slot single-layer 3-phase motor stator winding.

Despite the differences of poles number, slots number, and the coiling form of the 3-phase AC motor, the physical model of stator can be described as in Figure 1 for the symmetry of the magnetic circuit and the magnetomotive force (MMF) generated by powered winding. Figure 2 shows 

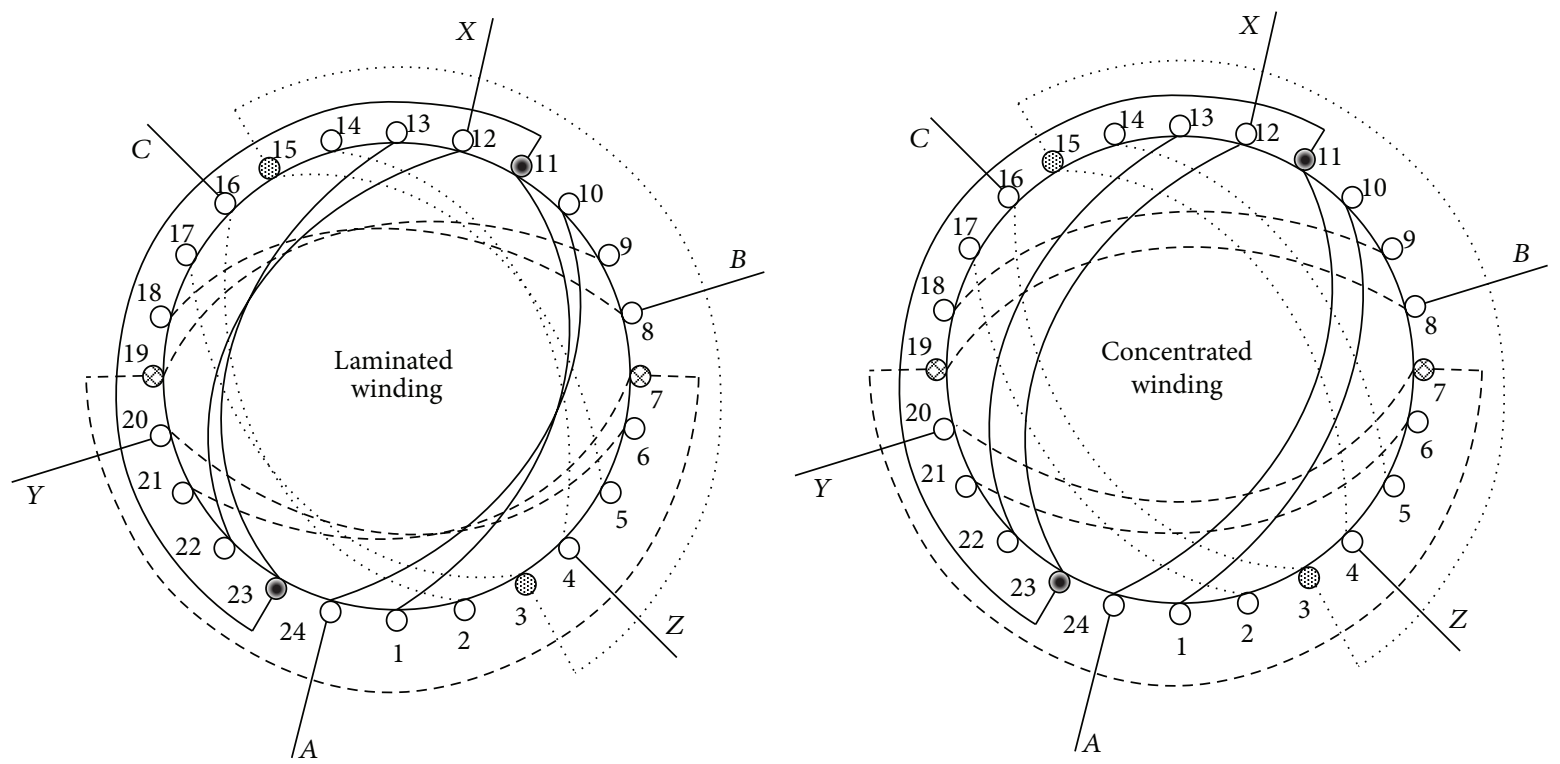

FIGURE 1: Distributed winding form.

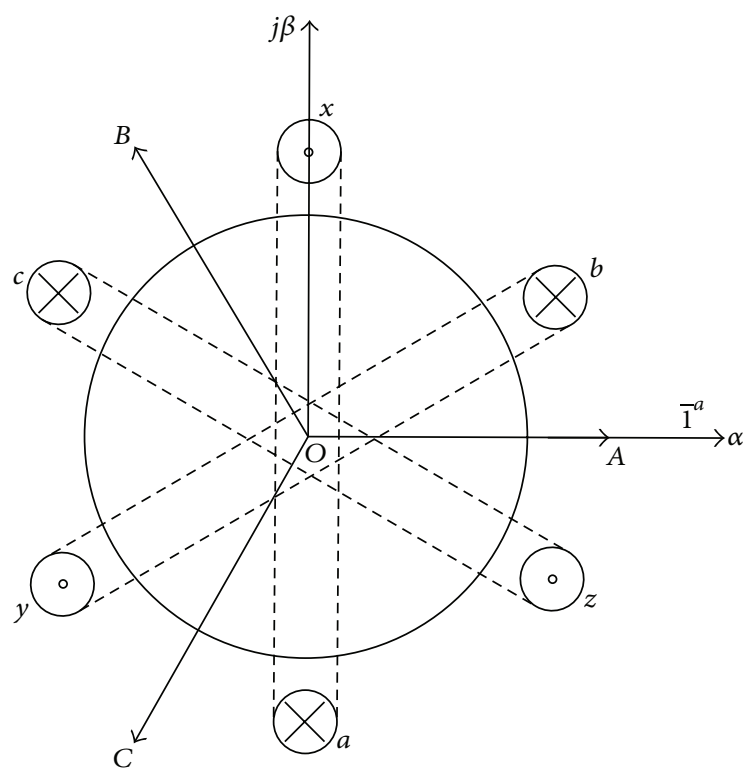

FIGURE 2: Simplified stator model of synchronic motor.

the distances of $2 \tau$ about a pair of magnetic poles equivalent to $360^{\circ}$ of electrical angle. Every stator of 3-phase AC motor can be analyzed with this model.

2.2. Circular Rotating Magnetic Field. When powering the stator model with the 3-phase current as (2.2), setting the positive direction from $a$ to $x, b$ to $y$, and $c$ to $z$, the 3 -phase MMF is generated which can be considered as sinusoidal distribution in the stator when excluding space harmonics. Then the MMF can be expressed as (2):

$$
\begin{gathered}
i_{a}=I_{m} \cos \omega t, \\
i_{b}=I_{m} \cos \left(\omega t-120^{\circ}\right),
\end{gathered}
$$

$$
\begin{gathered}
i_{c}=I_{m} \cos \left(\omega t+120^{\circ}\right), \\
\mathbf{F}_{a}(t)=0.5 \mathbf{F}_{a}\left(e^{j \omega t}+e^{-j \omega t}\right), \\
\mathbf{F}_{b}(t)=0.5 \mathbf{F}_{b}\left(e^{j \omega t} e^{-j 120^{\circ}}+e^{-j \omega t} e^{j 120^{\circ}}\right), \\
\mathbf{F}_{c}(t)=0.5 \mathbf{F}_{c}\left(e^{j \omega t} e^{j 120^{\circ}}+e^{-j \omega t} e^{-j 120^{\circ}}\right) .
\end{gathered}
$$

$\mathbf{F}_{a}$ is an MMF vector generated by the maximum current of A phase, the direction of which is assumed as the horizontal axis of static frame. $\mathbf{F}_{a}(t)$ is determined by $i_{a}$ varied with time t. $\mathbf{F}_{b}$ and $\mathbf{F}_{c}$ are similar to $\mathbf{F}_{a}$, which lead $\mathbf{F}_{a}$ by $120^{\circ}$ and $240^{\circ}$, respectively; $\mathbf{F}_{b}(t)$ and $\mathbf{F}_{c}(t)$ are with the same meaning of $\mathbf{F}_{a}(t)$.

The composite MMF in the air gap will be expressed as

$$
\Sigma \mathbf{F}(t)=\mathbf{F}_{a}(t)+\mathbf{F}_{b}(t)+\mathbf{F}_{c}(t)=1.5 \mathbf{F}_{a} e^{j \omega t} .
$$

It is a rotating MMF vector, of which the amplitude is 1.5 times of each phase. The electric angle of the MMF rotating in the space corresponds to that of the current changing in the winding, which is

$$
\theta=\omega t
$$

When the current changes by a cycle, the rotating MMF goes $2 \tau$ distances in the air gap. The revolution per second is

$$
n_{1}=\frac{f}{p_{\tau}},
$$

Where $f$ is the frequency of the stator current and $p_{\tau}$ is the number of pole pairs of the motor. 


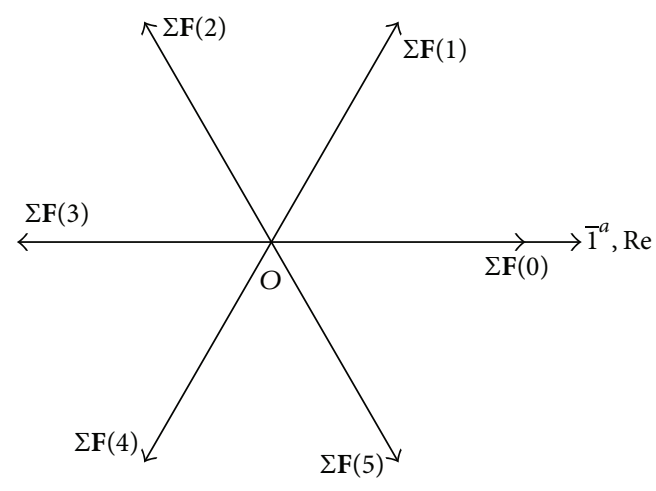

FIGURE 3: Stepping MMF of three-phase winding as $b_{H}=6$.

2.3. Discrete Magnetic Field and Positioning Torque. The MMF $\mathbf{F}_{s}$ generated by stator is to drive the rotor MMF $\mathbf{F}_{r}$ to rotate synchronously. The electromagnetic toque $T_{e}$ can be described in terms of $\mathbf{F}_{s}$ and $\mathbf{F}_{r}$ :

$$
T_{e} \propto\left|\mathbf{F}_{r} \times \mathbf{F}_{s}\right|=\mathbf{F}_{r} \mathbf{F}_{s} \sin \theta .
$$

The $\theta$ is the angle form $\mathbf{F}_{r}$ to $\mathbf{F}_{s}$. If $\mathbf{F}_{s}$ stops rotating at some position and $\mathbf{F}_{r}$ coincides with it, $\theta=0$, the electromagnetic toque will be equal to zero, which will be a positioning point.

If the motor is powered with the currents described in

$$
\begin{gathered}
i_{a}(k)=I_{m} \cos \frac{2 \pi}{b_{H}} k, \\
i_{b}(k)=I_{m} \cos \left(\frac{2 \pi}{b_{H}} k-120^{\circ}\right), \\
i_{c}(k)=I_{m} \cos \left(\frac{2 \pi}{b_{H}} k+120^{\circ}\right),
\end{gathered}
$$

where $b_{H}$ is the number of pulse distributor's beats per cycle, the composite MMF will stop at some point as the pulse number $k$ which is a positive integer not to change. When the next pulse emits, $k=k+1$, the composite MMF will go forward with a little angle just like a step. Then, the rotating MMF in the last section is discretized into stepping MMF [8] expressed in

$$
\Sigma \mathbf{F}(k)=1.5 \mathbf{F}_{a} e^{j\left(2 \pi / b_{H}\right) k} .
$$

An example as $b_{H}=6$ will illustrate the stepping MMF graphically.

Each MMF will generate a positioning point, and the torque driving the rotor MMF to approach this point is defined as positioning torque. Here, the angle is calculated by electric angle; the actual step number $b$ per revolution and the stepping angle $\alpha$ are expressed as the following formula with the number of pole pairs $p_{\tau}$ :

$$
\begin{gathered}
b=p_{\tau} b_{H}, \\
\alpha=\frac{360^{\circ}}{b}=\frac{360^{\circ}}{p_{\tau} b_{H}} .
\end{gathered}
$$

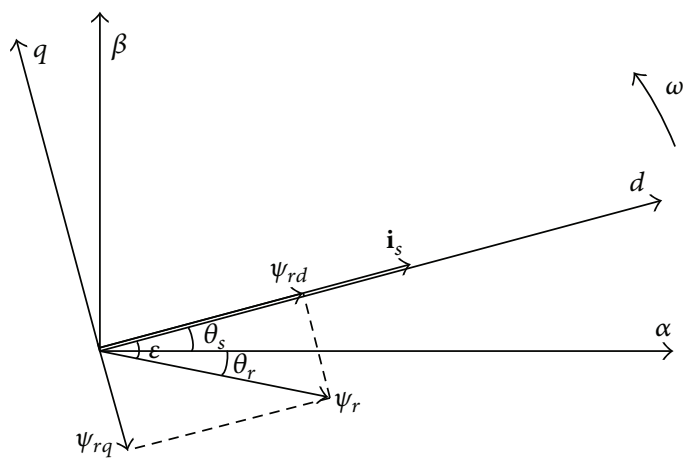

FIGURE 4: Vector diagram of stator current orientation.

The stepping angle is determined by $p_{\tau}$ and $b_{H}$. If one wants to increase the stepping number per revolution, it is better to increase $b_{H}$, since the number of pole pairs is constrained by motor structure.

\section{PMSM Model for Step Motion}

3.1. Motor Model by Stator Current Orientation. Make the angular speed of the rotating frame equal to that of stator current vector in general frame of PMSM which is shown in Figure 4 based on the $\alpha$ - $\beta$ static frame. The rotating frame is built by $\mathbf{i}_{s}$, the horizontal axis coinciding with $\mathbf{i}_{s}$ is named $d$ axis, and the vertical axis orthogonal to $d$-axis is $q$-axis. Then, general frame becomes the $d-q$ frame orientated by stator current [9]. In the figure, the angle from $\psi_{r}$ to $\mathbf{i}_{s}$ is assumed as $\varepsilon$, and $\theta_{s}$ and $\theta_{r}$ represent the angle form $\alpha$-axis to $\mathbf{i}_{s}$ and $\psi_{r}$, respectively. $\omega$ is the angular speed of the rotating frame.

The two components of $\mathbf{i}_{s}$ in the frame, named $i_{s d}$ and $i_{s q}$, are expressed as

$$
\begin{gathered}
i_{s d}=i_{s}=\left|\mathbf{i}_{s}\right|, \\
i_{s q}=0 .
\end{gathered}
$$

According to the mathematical expression of PMSM on rotating frame, the flux function can be rewritten as the following equation:

$$
\begin{gathered}
\psi_{s d}=L_{d} i_{s d}+\psi_{r d}=L_{d} i_{s}+\psi_{r d}, \\
\psi_{s q}=L_{q} i_{s q}+\psi_{r q}=\psi_{r q},
\end{gathered}
$$

where $\psi_{s d}$ and $\psi_{s q}$ are $d-q$ components of stator flux in rotating frame, $\psi_{r d}$ and $\psi_{r q}$ are $d-q$ components of rotor flux, and $L_{d}$ and $L_{q}$ are $d-q$ components of stator selfinductance. The torque function can be expressed as the following formula with (10) and (11):

$$
\begin{aligned}
T_{e} & =p_{\tau}\left|\psi_{s} \times \mathbf{i}_{s}\right| \\
& =p_{\tau} \psi_{s d} i_{s q}-p_{\tau} \psi_{s q} i_{s d} \\
& =-p_{\tau} \psi_{r q} i_{s} .
\end{aligned}
$$

Substituting $\sin (-\varepsilon)=\psi_{r q} / \psi_{r}$ into (12), the electromagnetic torque function can be rewritten as

$$
T_{e}=p_{\tau} i_{s} \psi_{r} \sin \varepsilon
$$


$\varepsilon$ is also defined as torque angle; when it is greater than zero, with $\psi_{r}$ being drawn by $\mathbf{i}_{s}$, the electromagnetic torque is positive.

3.2. Structure of the Control System. Unlike the VC and DTC, in this control method, magnitude and phase of stator current are regulated dynamically for best torque responding, instead of keeping the amplitude of stator current and rotor flux or maintaining the angle $\varepsilon$ between the current and the flux equal to $90^{\circ}$. Because the rotor flux is unchanged, the regulable variables of the control system are no other than the magnitude of stator current $\left|i_{s}\right|$ and the angle $\varepsilon$.

The structure of motor control system can be simplified as shown in Figure 5 which includes an inner loop and an outer loop.

The outer loop is the only one closed loop to control the speed or position. In the loop, the input is the rotor angle frequency difference or angle difference of preset and feedback, and the output is preset current vector including the magnitude and the rotation angle. To regulate the two variables, we give the motor the maximum current for maximum torque to start or brake and supply the rated current and adjust the $\varepsilon$ to change the electromagnetic torque when the motor operates steadily.

The inner loop is current loop, in which the three-phase stator current is transformed into current vector on $d$ - $p$ frame and the vector is compared with the preset current vector from the previous regulator. The difference of the current vector is to select the voltage vector for inverter control. It can use the method of direct current control (DCC) in [10], which follows the synchronized on-off principle. The current vector at every time interval is predicted for two possible cases as the following formula:

$$
\begin{aligned}
\mathbf{i}_{\alpha, \beta}(k+1)= & \underbrace{\mathbf{i}_{\alpha, \beta}(k)\left(1-\left(T / T_{s}\right)\right)}_{\mathbf{i}_{0(\alpha, \beta)}(k+1)} \\
& +\underbrace{\left(T / L_{s}\right) \mathbf{u}_{\alpha, \beta}(k)}_{\mathbf{i}_{u(\alpha, \beta)}(k+1)},
\end{aligned}
$$

where $\mathbf{i}_{0}$ is the radial naturally decreased current vector, $\mathbf{i}_{u}$ is the applied current vector generated by constant voltage during the sampling interval, and the subscripts $\alpha$ and $\beta$ represent the vector components of static frame. The voltage vector $\mathbf{u}$ at instant $k$ can take the following value by decomposing on static $\alpha-\beta$ frame:

$$
\begin{aligned}
u_{\alpha, \beta}(k) & =U_{\mathrm{DC}}\left[\begin{array}{l}
K_{U \alpha}(k) \\
K_{U \beta}(k)
\end{array}\right] \\
& =U_{\mathrm{DC}}\left[\begin{array}{ccc}
\frac{2}{3} & -\frac{1}{3} & -\frac{1}{3} \\
0 & \frac{1}{\sqrt{3}} & -\frac{1}{\sqrt{3}}
\end{array}\right]\left[\begin{array}{l}
s_{T 1} \\
s_{T 2} \\
s_{T 3}
\end{array}\right] .
\end{aligned}
$$

$U_{\mathrm{DC}}$ is the DC-link voltage. $s_{T 1}, s_{T 2}$, and $s_{T 3}$ denote the states ( 0 or 1 ) of upper transistors in the inverter, which include six effective vectors $(100,110,010,011,001,101)$ and two zero vectors $(000,111)$. After calculating $i_{0}$, the six voltage vector closest to the direction of the error between $i_{s}^{*}$ and $i_{s}$ is chosen. Figure 6 shows the particular case of selecting upper transistors 010 .

3.3. Discrete Current Control. When the stator is powered with the discrete current as (7), the stator current vector $\mathbf{i}_{s}$ has $b_{H}$ positioning points at the stator circle shaping a regular polygon MMF shown in Figure 3, for example,

$$
\mathbf{i}_{s}=\frac{3}{2} I_{m} e^{j\left(2 \pi / b_{H}\right) k}
$$

The angle between the two adjacent current vectors is defined as stepping angle just like the step motor, which is

$$
\theta_{b}=\frac{2 \pi}{b_{H}} .
$$

Therefore, the torque of PMSM can be written as

$$
T_{e}=T_{\max } \sin \left(k \theta_{b}-p_{n} \alpha\right),
$$

where $\alpha$ is the mechanical angle of rotating and $p_{n}$ is the number of pole pairs.

This torque is also called reposition torque, impelling the rotor to run forward to catch up with the stator. Therefore, the stopping point of the stator current vector is the very positing point achieving incremental movement of a motor. Take $b_{H}=$ 12 and $T_{Z}=0$ (motor idling), for example, so the discrete current vector and the position are shown in Figure 7.

The proposed strategy of PMSM is called discrete current control, in which the main control variable is the torque angle between stator current vector and rotor flux vector, and the amplitude of stator current is the rating (except for starting and braking which is the maximum). It is different from VC and DTC, and the latter is to control the angle of flux of stator and rotor keeping the stator flux constant. The proposed strategy is more suitable for positioning because of the characteristic of positioning torque generated by discrete current and stepping motion, and the control process is also easier than the two classical methods.

\section{Discrete Current Control of PMSM}

To describe the proposed control strategy, two errors generated in the operation must be declared.

(1) Static angle error: generated by load torque. It needs an electromagnetic torque to balance, so the torque angle cannot be decreased to zero which become an error for the control.

(2) Dynamic angle error: the following process of rotor is not synchronous with stator current vector. The rotor will lag behind the vector when driving or go beyond the positioning point when braking. But the dynamic error will be disappeared when the rotor stops.

4.1. Pointing Control. Pointing control is a typical discrete control method, controlling the motor to move a step forward every time. Only when the transient process of the first step is completely terminated, the second step begins. 


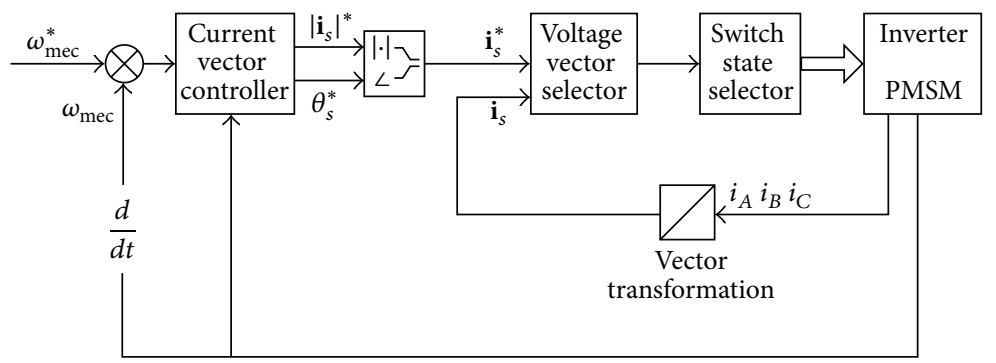

FIGURE 5: Block diagram of stator current oriented control system PMSM.

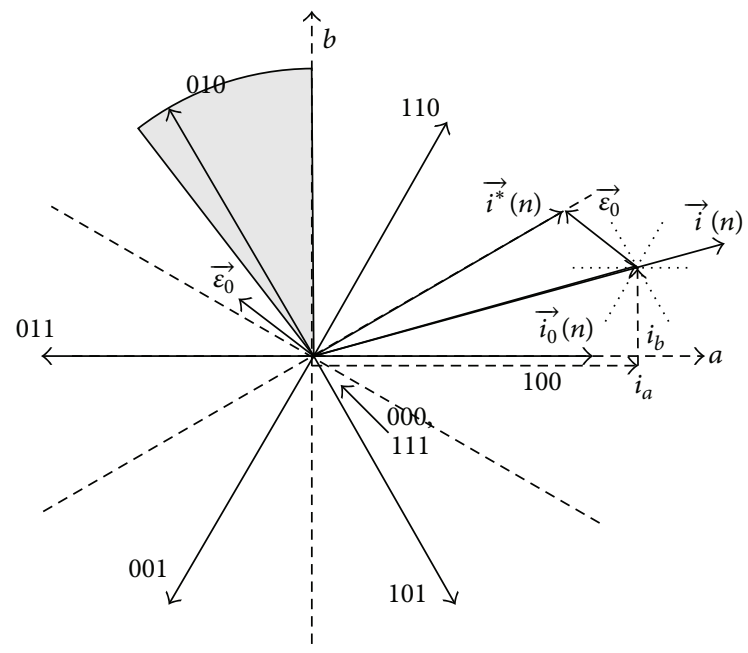

FIGURE 6: Current vector regulation based on voltage space vector.

The one-step torque $T_{b}$ should be greater than static load torque, so that the static angle error can be less than a stepping angle. The dynamic angle error, for example, should be less than $150^{\circ}$ to keep the operation not losing its step when $b_{H}=$ 12. The angle of one step is $k \theta_{b}$; the minimum one is $\theta_{b}$ and the maximum one must be less than the dynamic angle error.

The greater the stepping angle, the more serious the oscillation phenomenon near the positing point, which needs to be avoided if possible. The simulation result is shown in Figure 8 . The motor is triggered by the step pulse every 0.4 seconds with the rise time of $0.025 \mathrm{~s}$ and the overshoot of about $32 \%$. The rotor stopped at the given point after the second oscillation.

The oscillation of pointing control is produced by $\Delta \theta=$ $k \theta_{b}-p_{n} \alpha$, and $\omega$ is not equal to zero at the same time, and the torque near the positing point will be so small. These problems can be solved with "bang-bang control" of optimal time and maximum torque.

(1) The time-optimal method is to brake at a proper time to remove the overshoot. As shown in Figure 9, the preset current vector angle is $\theta_{s}=-\theta_{b}=30^{\circ}$; then the rotor accelerated for $\varepsilon$ which is equal to $\theta_{b}$ when $t=0$. When $t=0.018 \mathrm{~s}$, the vector was back to $0^{\circ}$ and $\varepsilon<0$, and the motor began to decelerate. When $t=0.026 \mathrm{~s}, \theta_{s}=k \theta_{b}=\theta_{r}=p_{n} \alpha$, and $\omega=0$, the vector was set at $\theta_{s}=30^{\circ}$ again and the rotor stopped at the

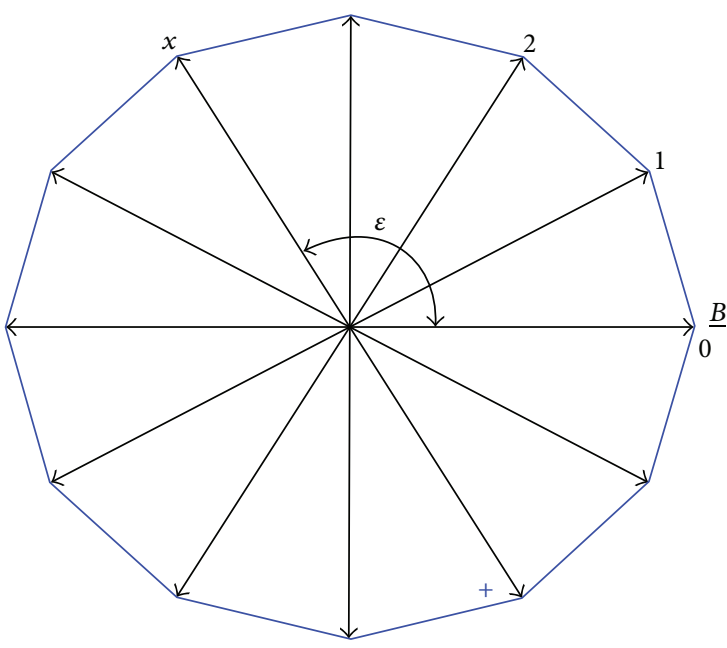

FIGURE 7: Positioning star diagram.

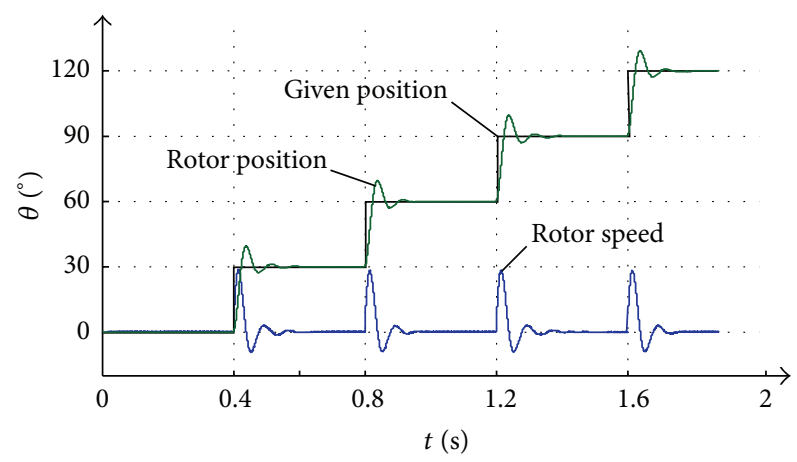

FIGURE 8: Position and speed curve under point control.

positioning point. In the process, the transient time is 0.031 , which decreases to its $1 / 6$.

(2) Maximum torque control is to give the maximum torque at the accelerating stage and brake with the maximum negative torque when the position is vicinity to the stator current vector. The maximum torque is generated as $\varepsilon=90^{\circ}$. In the simulation shown as Figure 10, transforming time of the vector is at $t_{1}=0.010 \mathrm{~s}$ and $t_{2}=0.017 \mathrm{~s}$. Before $t_{1}$, let $k=3$ and after it $k=-2$, and at $t_{2}$, make $k=1$ to keep the rotor stable. In this control, the transient time is only $0.027 \mathrm{~s}$, which decreases to $1 / 8$ of the original time. 


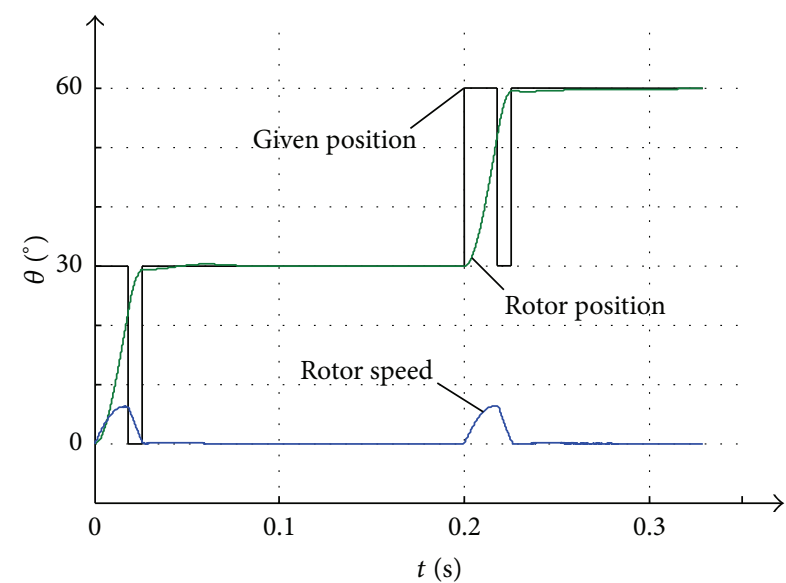

FIGURE 9: Position and speed curve under time optimization.

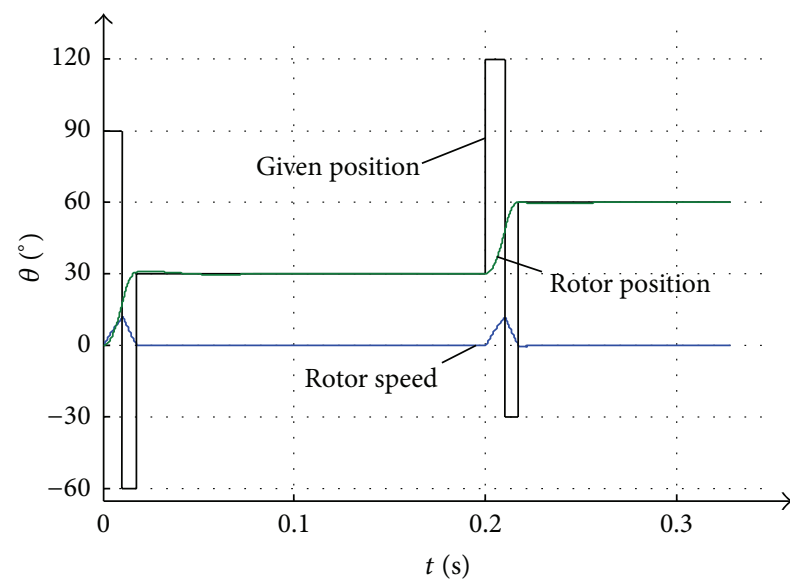

Figure 10: Position and speed curve under maximum torque.

4.2. Constant Frequency Control. Some motors need a constant frequency control method, which is only to change the step number in a constant frequency and to keep it not losing its steps. The angle frequency of motor $\omega_{r}$ will follow the given frequency $\omega_{s}$ by $\varepsilon$ which must be less than $180^{\circ}$. After a bit oscillations, the rotor will reach the state of $\omega_{r}=\omega_{s}$, while the given frequency has a maximum critical value named jumping frequency, which is defined as the highest frequency so that the motor does not lose its step. If $\omega_{s}$ is more than the jumping frequency, $\omega_{r}$ cannot catch up with $\omega_{s}$ and the position of rotor will lag behind the stator current vector, which will lead to a serious fault.

In the positioning control of this method, the motor responses will oscillate in starting and braking time. These oscillations can be eliminated by optimal controls as which is used in pointing control. The response curves generated by this method will be shown in the experiment in Section 5.

4.3. Up-Down Frequency Control. It needs more time to accelerate or decelerate for the large-capacity motor, because the rotor could store more kinetic energy. If only give the motor a step change in constant frequency, the dynamic angle error may be over the maximum and lead to steps losing.

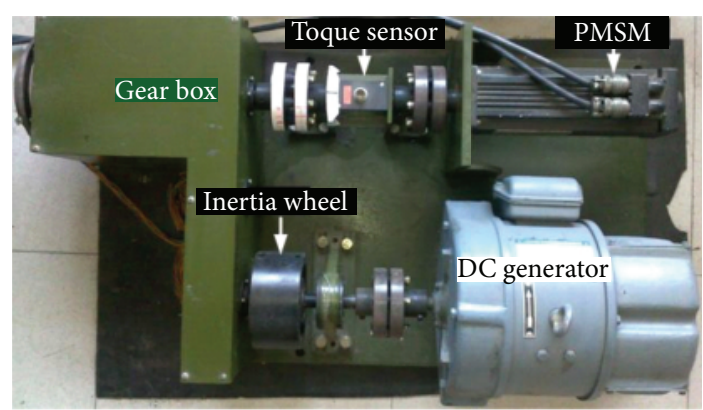

FIGURE 11: Experiment platform.

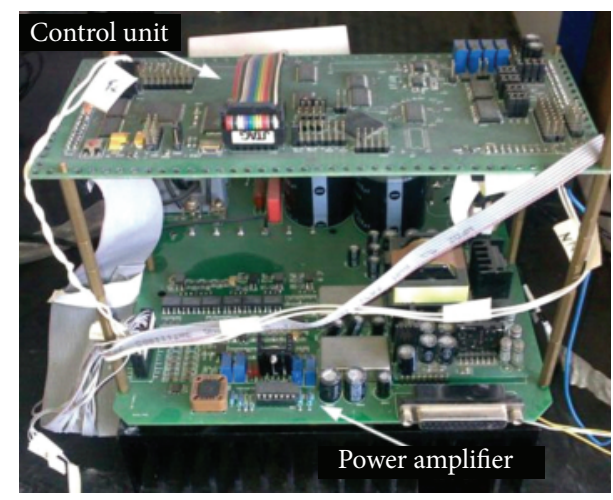

FIGURE 12: Digital driving controller.

It is necessary to preset an increment or decrement frequency of the motor to accelerate or decelerate.

The highest frequency is limited by the electromagnetic torque which is a function of angle frequency. A frequency of stator current vector, which is less than the jumping frequency, is given to accelerate at $t=0(+)$. Then the frequency increases gradually and the time interval of every step decreases. The $\varepsilon$ had better to be control in the range of $90^{\circ} \pm \theta_{b}$ to maintain the maximum torque and not to lose its step.

Generally, to obtain a better result of control, this control is designed with closed loop to get an optimal up frequency curve. Moreover, the curve of frequency will be designed as two, three, or five segments according to the travel length. The experiment of three-segment curve is shown in Section 5.

\section{Experiments}

The experiments are based on a device of PMSM, which includes motor and transmission platform and digital driving controller. The platform is shown in Figure 11. The PMSM is of the type of M205B produced by KOLLMONGEN in US with rated power of $1.6 \mathrm{~kW}$, rated voltage of $230 \mathrm{~V}$, continuous rated current of $5.3 \mathrm{~A}$, continuous torque of $4.47 \mathrm{Nm}$, and maximum revolution of $3600 \mathrm{rpm}$. The load is a DC generator with $1.1 \mathrm{~kW}$ rated power and the transmission ratio is $1: 1$ of the gear box. The connecting mechanism between the two motors is with toque sensor, harmonic reducer, 


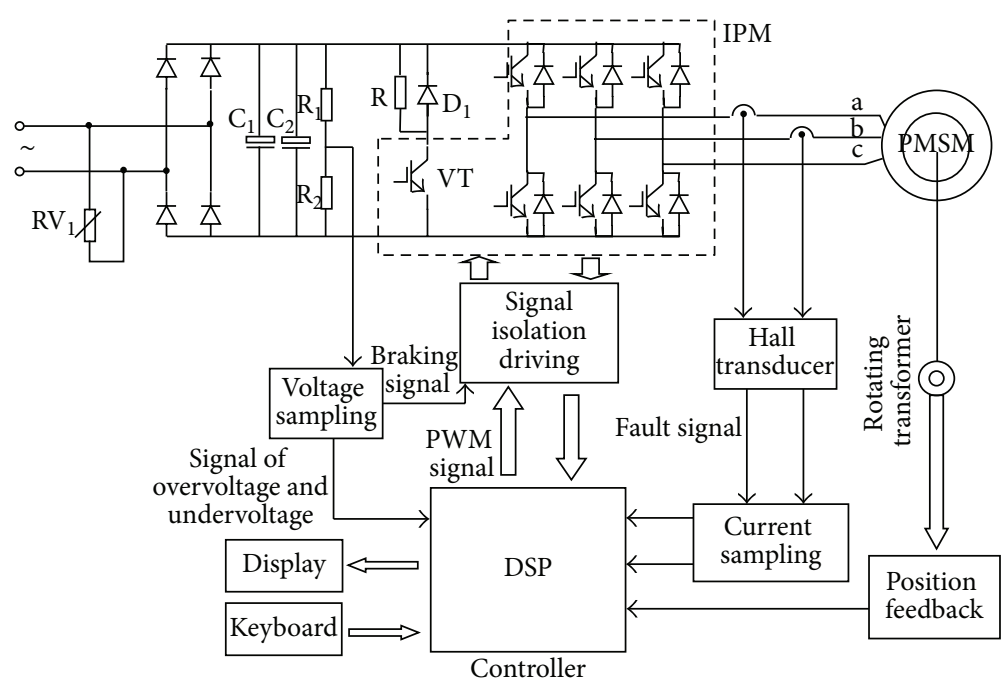

FIgURE 13: The structure diagram of control system.

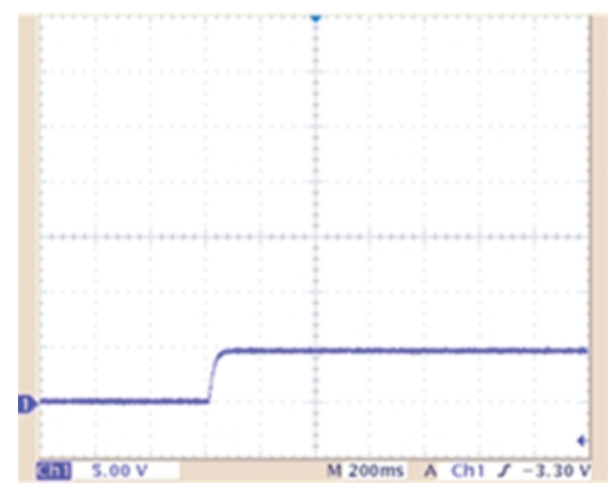

(a) Current change of A phase

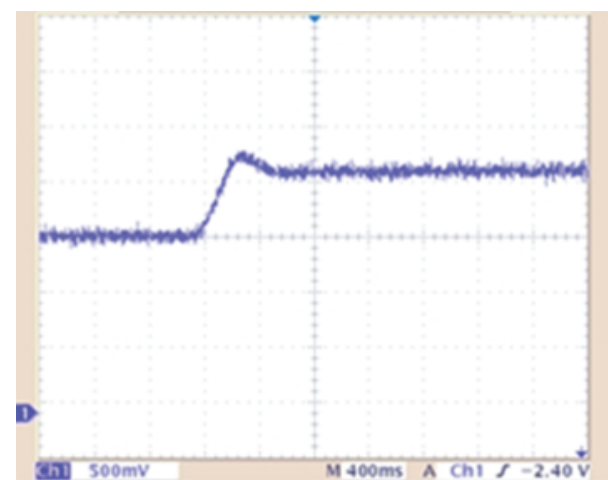

(b) Position curve

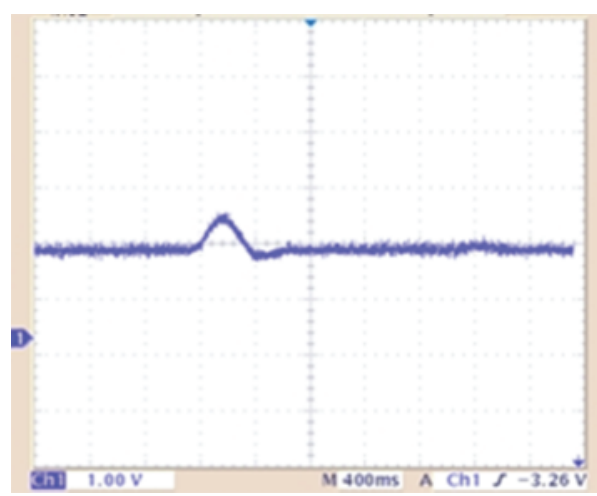

(c) Speed curve

FIGURE 14: Experiment curve of pointing control.

and inertia wheel. The application of PMSM can be well approximated by these devices.

The digital driving controller is composed of control unit and power amplifier shown in Figure 12. The kernel of control part is a TMS320F240 chip of DSP produced by TI and around it are the peripheral circuit and $\mathrm{A} / \mathrm{D}$ circuit. The main part of power amplifier is PM15RSH120, which is a intelligent power module (IPM) produced by Mitsubishi. Beside the IPM, the accessory circuit includes trigger signal driver circuit, special power supply module of JS158, position detecting circuit, current sampling circuit, and protection circuit.

The structure diagram of the control system is shown in Figure 13. 


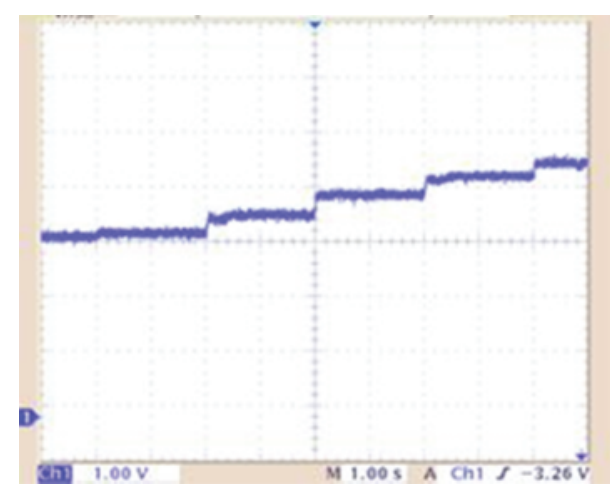

(a) Current change of A phase

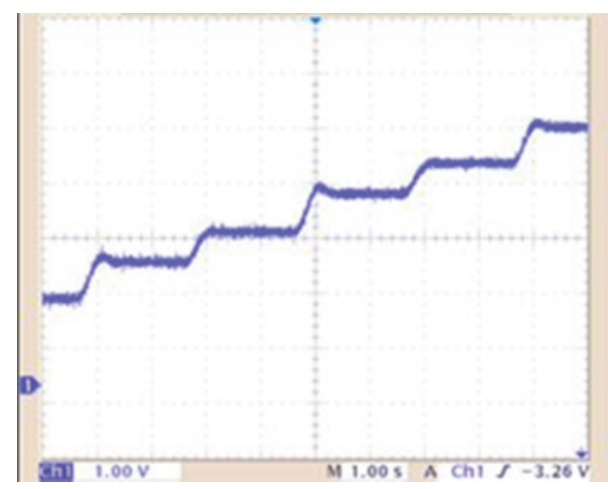

(b) Position curve

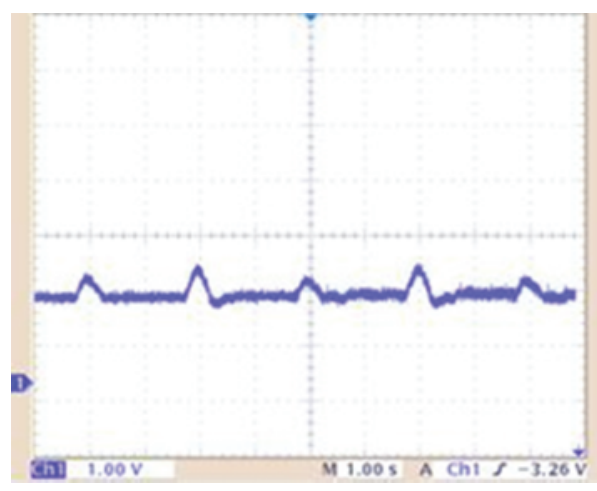

(c) Speed curve

FIGURE 15: Experiment curve of constant frequency control.

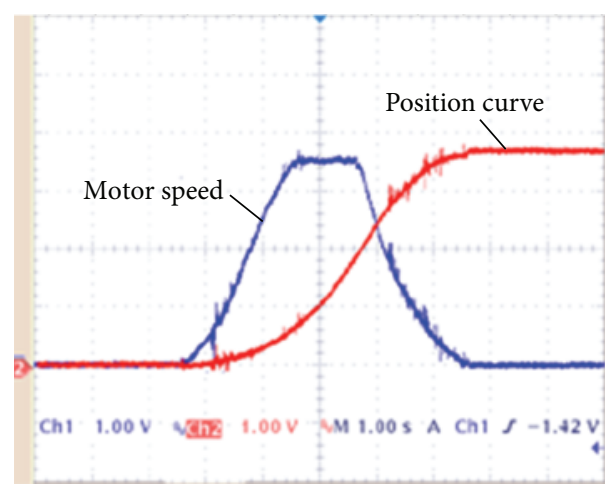

(a) Position and speed curve

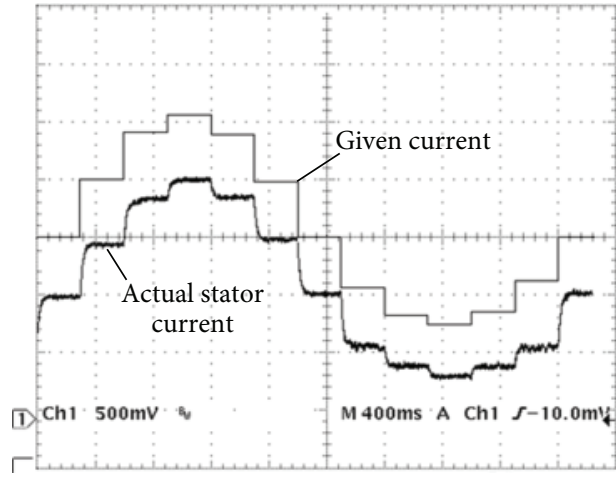

(b) Current of A phase

FIGURE 16: Experiment curve of up-down frequency control.

5.1. Control Curve. In the experiment, the motor is with 2 pairs of pole and the electric angle is $720^{\circ}$ per revolution. We divided the cycle of stator current into 12 parts and the electric angle will be $30^{\circ}$ per step. The number of positioning point will be $12 \times 2$ per revolution and every step is corresponded to $15^{\circ}$.

5.1.1. Pointing Control. The stator current vector is given as formula (7). When $t=0, k=0$ and the motor stays at the initial position. When $t=0.6 \mathrm{~s}$, let $k=1$; the vector will lead the rotor flux by a stepping angle that is equal to $30^{\circ}$ and the rotor will follow the vector by the reposition torque.
The current change of A phase is shown in Figure 14(a) and the responded curve of position and speed is in Figures 14(b) and $14(\mathrm{c})$.

5.1.2. Constant Frequency Control. In order to watch the control process, this experiment uses a frequency of $0.5 \mathrm{~Hz}$. From Figure 15, the rotor position is following the stator current vector closely and the positioning performance is obvious in the discrete control.

5.1.3. Up-Down Frequency Control. Three-segment-speed curve of motor is used in rapid positioning, which only 
TABLE 1: Experiment data of position precision incensement motion.

\begin{tabular}{lcccccc}
\hline Given step & 1 & 12 & 24 & 100 & 500 & 1000 \\
Pulse number & 181 & 2052 & 4224 & 17002 & 85452 & 170702 \\
Rotating angle & $15.9^{\circ}$ & $180.35^{\circ}$ & $371.25^{\circ}$ & $1494^{\circ}$ & $7510.4^{\circ}$ & $62464.02^{\circ}$ \\
Actual step & 1.1 & 12.02 & 12.7 & 99.6 & 500.7 & 1000.2 \\
\hline
\end{tabular}

TABLE 2: Experiment data of operating 160 revolutions.

\begin{tabular}{lccccc}
\hline $\begin{array}{l}\text { No. } \\
\text { Distance (pulse }\end{array}$ & 1 & 2 & 3 & 4 & 5 \\
$\begin{array}{l}\text { number) } \\
\text { Time (s) }\end{array}$ & 655202 & 655365 & 655369 & 655406 & 655485 \\
$\begin{array}{l}\text { Error (pulses } \\
\text { number) }\end{array}$ & 158 & 5 & 9 & 46 & 125 \\
\hline
\end{tabular}

includes accelerating, constant speed, and decelerating. The experiment curve is shown in Figure 16(a) and the positioning accuracy is limited below a stepping angle. The currentfollowing curve is shown in Figure 16(b), in which the actual current curve is moved down a division of oscilloscope for watching clearly.

5.2. Analyses. Analyzing the error of stepping control of PMSM, we can gain the precision of it used in positioning. The steady error is less than one stepping angle which is $15^{\circ}$ here. If we use the pulses of rotary encoder, of which $360^{\circ}$ is corresponded to 4096 pulses, to stand for the absolute position, we can get a table of precision.

When driving the motor to run 160 revolutions, the emitting pulses and the operation time are shown in Tables 1 and 2 .

If use open loop control method and let the speed follow the three-segment curve, when the rotor moves 160 revolutions, then the number of pulses is 655360 , and we get the result recorded in Table 2 .

It is proved that the discrete current vector method of PMSM has more advantages than existing methods. Firstly, the structure is simply just using single loop. Secondly, the control method with discrete MMF can generate the larger torque to start or drive the high inertia loads. Thirdly, positioning precision is determined by the stepping angle that can get higher accuracy. Moreover, the reliability and robustness of this method are better than those of the original driver which needs to often change its parameter especially for high inertia loads.

\section{Conclusion}

In this paper, a stepping control method of PMSM is presented. In the method, the circle of rotating MMF is discretized to regular polygon, and in this case, the positioning on stator current orientation has been discussed with the mechanism model of PMSM. The three methods of control are simulated and tested in experiment, which is available with a general DSP controller.
Although good performance is achieved, the method needs deeper studies in theory and applications, such as current responding, harmony wave analysis of discrete current, and influence of the method to grid. Our further works in this area will be oriented to implementation of this method in transmission technology of valve and artillery in order to improve the performance and efficiency and simplify structure.

\section{Acknowledgments}

This work is supported by the Natural Science Funds of Hebei Province (E2013202108) and by the National High Technology Research and Development Program of China (863 Program) (2006AA040306).

\section{References}

[1] T. F. Blaschke, "Das Prinzip der Feldorientierung, die Grundlage fur die TRANSVEKTOR-Regelung von Asynchronmaschinen," Siemens Zeitschrift, vol. 45, no. 10, pp. 757-760, 1971.

[2] A. B. Nikolic and B. I. Jeftenic, "Precise vector control of CSI fed induction motor drive," European Transactions on Electrical Power, vol. 16, no. 2, pp. 175-188, 2006.

[3] M. Depenbrock, "Direkte selbstregelung, (DSR) für hochdynamische mit umrichtersptisun," ETZ-Archiv, vol. 7, no. 7, pp. 211-216, 1985.

[4] S. M. Gadoue, D. Giaouris, and J. W. Finch, "Artificial intelligence-based speed control of DTC induction motor drives-a comparative study," Electric Power Systems Research, vol. 79, no. 1, pp. 210-219, 2009.

[5] Z. Sorchini and P. T. Krein, "Formal derivation of direct torque control for induction machines," IEEE Transactions on Power Electronics, vol. 21, no. 5, pp. 1428-1436, 2006.

[6] L. Yituo, L. Haifeng, Q. Wenlong et al., "A novel initial rotor position estimation method for permanent magnet synchronous motors," Proceedings of the Chinese Society for Electrical Engineering, vol. 33, no. 5, pp. 75-82, 2013.

[7] M. Oettmeier, M. Spichartz, V. Staudt, and A. Steimel, "Statorflux-oriented control of PMSM in traction-Experimental results," in Electrical Systems for Aircraft, Railway and Ship Propulsion (ESARS '12), pp. 1-6, Bologna, Italy, 2012.

[8] Y. Yan, D. Yan, and C. Mingjun, "Research on application of PMSM step motion control technology in weapon location system," Journal of Gun Launch \& Control, no. 3, pp. 19-23, 2008.

[9] D. Yan, S. Hexu, B. Zhiyuan, and W. Wei, "Permanent magnet synchronous motor position control system based on torqueangle control," Transactions of China Electrotechnical Society, vol. 21, no. 1, pp. 86-91, 2006.

[10] V. Ambrožič, R. Fišer, and D. Nedeljković, "Direct current control-a new current regulation principle," IEEE Transactions on Power Electronics, vol. 18, no. 1, pp. 495-503, 2003. 


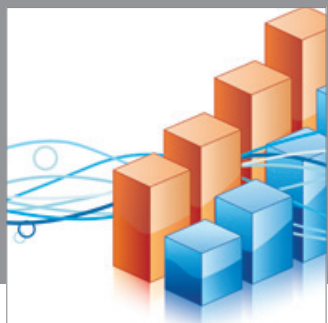

Advances in

Operations Research

mansans

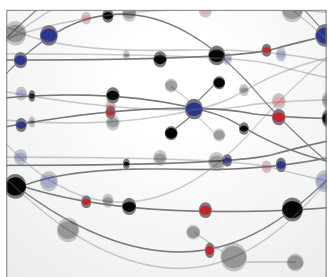

The Scientific World Journal
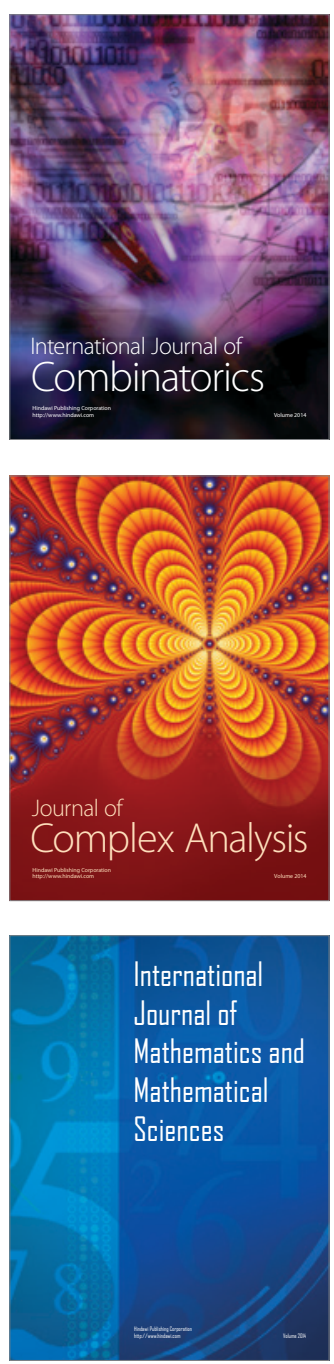
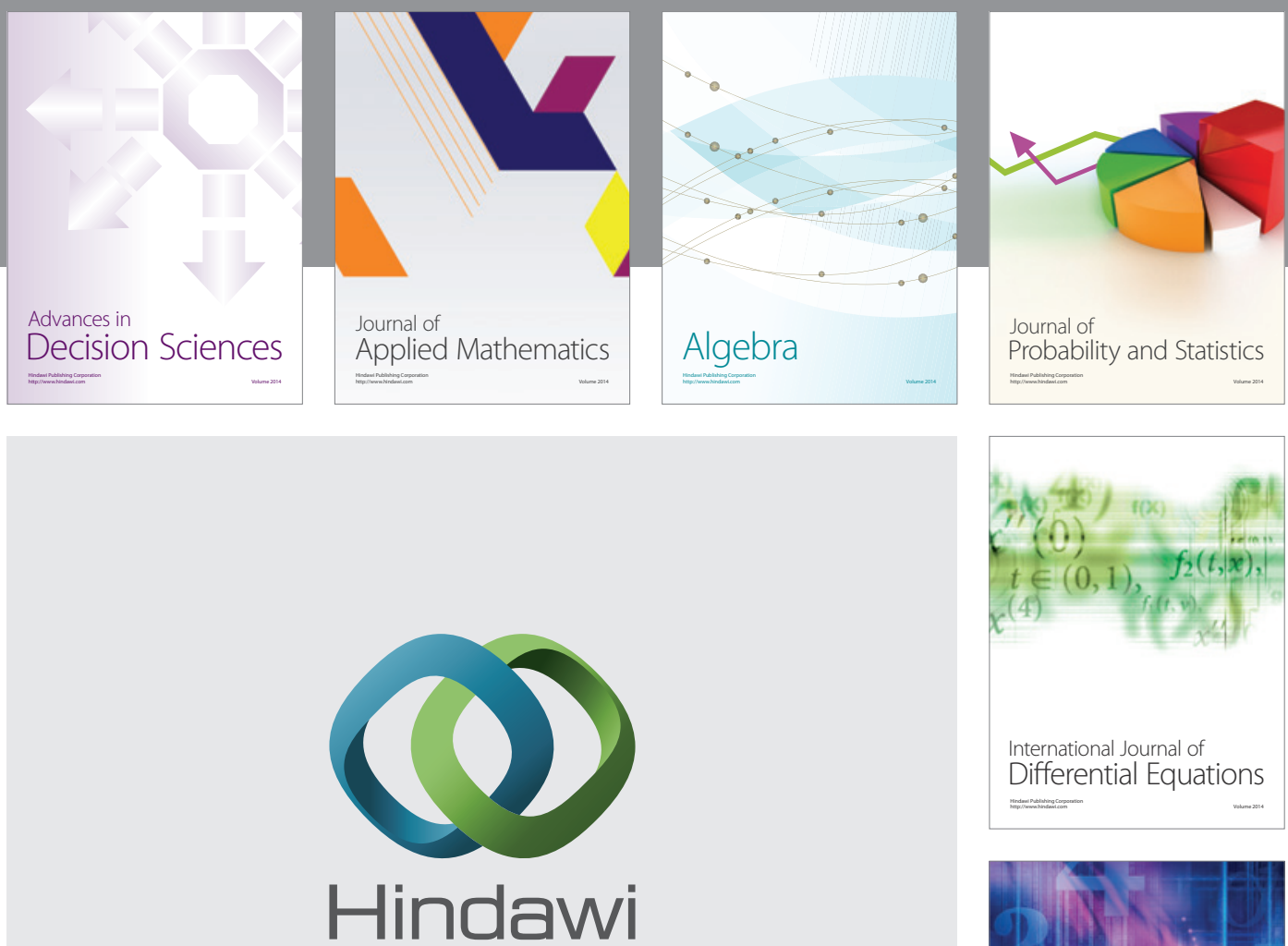

Submit your manuscripts at http://www.hindawi.com
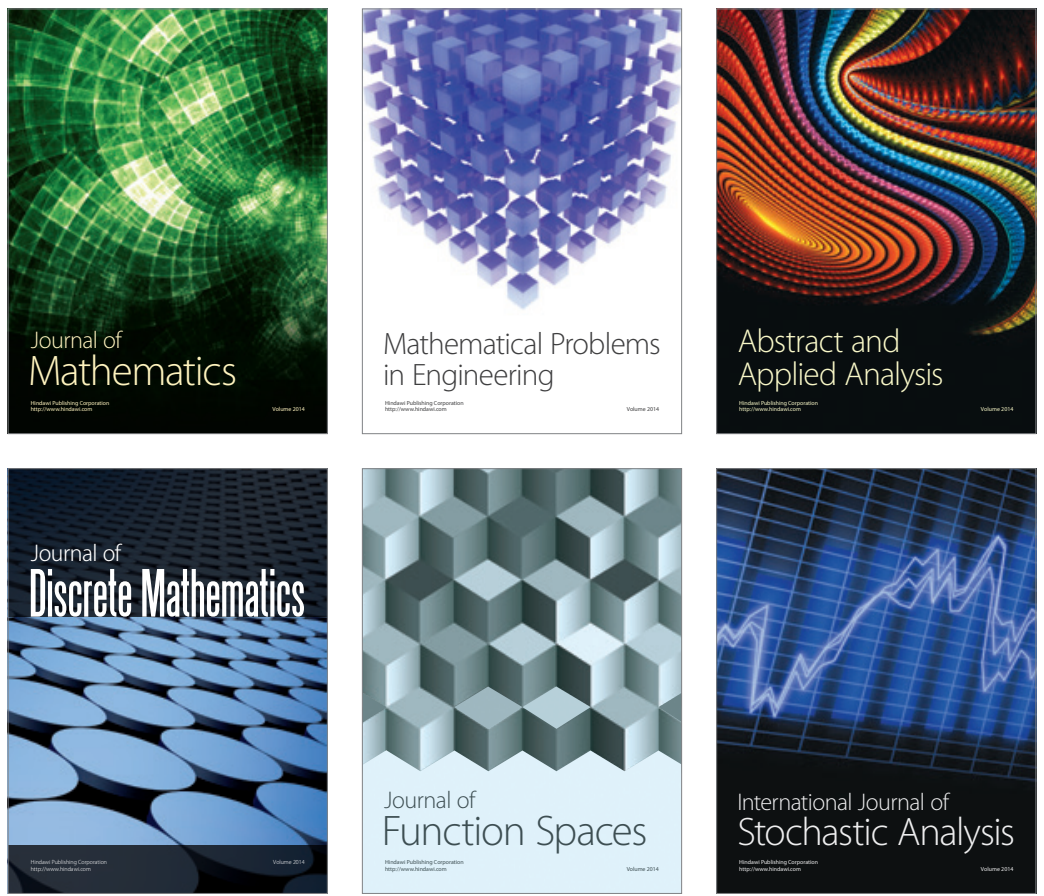

Journal of

Function Spaces

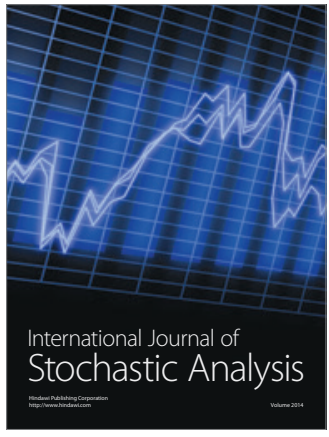

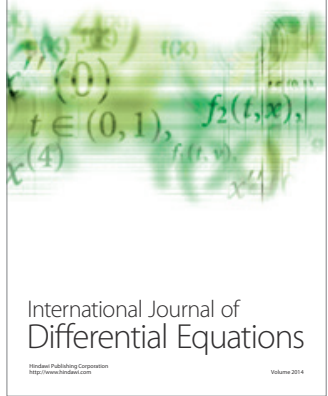
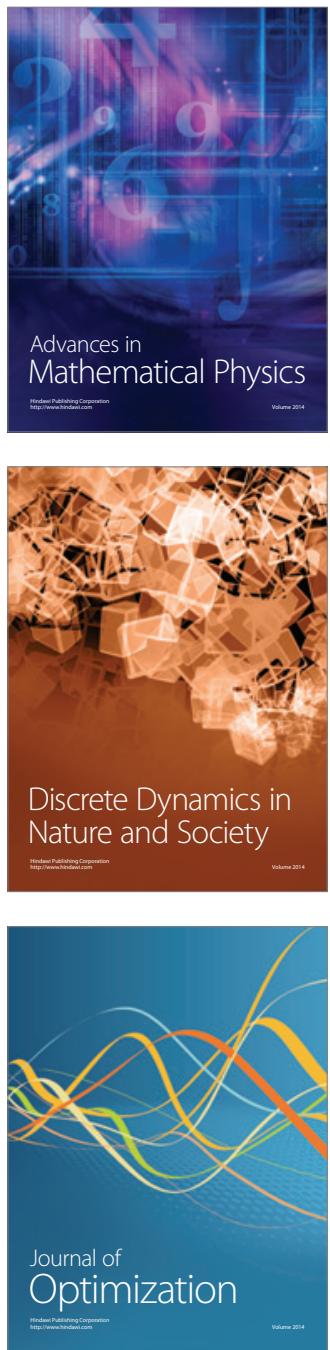